Mesure et évaluation en éducation

\title{
L'évaluation des compétences professionnelles : apports croisés de la littérature en évaluation, en éducation et en psychologie du travail
}

\section{Claire Tourmen}

Volume 38, numéro 2, 2015

URI : https://id.erudit.org/iderudit/1036765ar

DOI : https://doi.org/10.7202/1036765ar

Aller au sommaire du numéro

Éditeur(s)

ADMEE-Canada - Université Laval

ISSN

0823-3993 (imprimé)

2368-2000 (numérique)

Découvrir la revue

Citer cet article

Tourmen, C. (2015). L'évaluation des compétences professionnelles : apports croisés de la littérature en évaluation, en éducation et en psychologie du travail. Mesure et évaluation en éducation, 38(2), 111-144.

https://doi.org/10.7202/1036765ar

\section{Résumé de l'article}

Cette recension propose de synthétiser les apports croisés de la littérature en évaluation, en éducation et en psychologie du travail sur la question de l'évaluation des compétences professionnelles en formation. En effet, s'il s'agit d'une question nouvelle et d'actualité dans le champ de la formation, nous verrons que des auteurs ont proposé des définitions et des cadres pour modéliser des compétences, tandis que d'autres ont fait avancer la connaissance de l'activité des évaluateurs dans ces situations, ce qui permet d'en tirer certains enseignements. D'autres encore ont étudié les effets positifs mais aussi pervers de ces pratiques. Tout ceci constituera une revue de littérature qui pourra intéresser les praticiens, les enseignants et les formateurs professionnels désireux de connaître les avancées de la recherche sur ces questions. 


\title{
L'évaluation des compétences professionnelles: apports croisés de la littérature en évaluation, en éducation et en psychologie du travail
}

\author{
Claire Tourmen \\ Institut national supérieur des sciences agronomiques, de l'alimentation \\ et de l'environnement de Dijon (AgroSup Dijon)
}

Mots clés : évaluation, compétences, expérience, performance, activité

Cette recension propose de synthétiser les apports croisés de la littérature en évaluation, en éducation et en psychologie du travail sur la question de l'évaluation des compétences professionnelles en formation. En effet, s'il s'agit d'une question nouvelle et d'actualité dans le champ de la formation, nous verrons que des auteurs ont proposé des définitions et des cadres pour modéliser des compétences, tandis que d'autres ont fait avancer la connaissance de l'activité des évaluateurs dans ces situations, ce qui permet d'en tirer certains enseignements. D'autres encore ont étudié les effets positifs mais aussi pervers de ces pratiques. Tout ceci constituera une revue de littérature qui pourra intéresser les praticiens, les enseignants et les formateurs professionnels désireux de connaître les avancées de la recherche sur ces questions.

Keywords: evaluation, competencies, experience, performance, activity

In this review of literature, we will see how the question of professional competencies' evaluation has been developed in the literature in evaluation, in education and in psychology of work. It is indeed a new matter of concern in the field of education. We will see that authors have proposed definitions and frameworks to develop models of competencies, while others have gained knowledge on how evaluators evaluate competencies in real settings. Others have studied the positive but also the perverse effects of these practices. This review of literature may interest practitioners, teachers and professional trainers who are curious about research findings on these matters. 
Palavras-chave: avaliação, competências, experiência, desempenho, atividade

Esta recensão propõe-se sintetizar, de uma forma cruzada, os contributos da literatura em avaliação, educação e psicologia do trabalho sobre a questão da avaliação das competências profissionais em formação. Com efeito, tratando-se de uma questão nova e actual no domínio da formação, uns autores propuseram definições e quadros para modelizar as competências, enquanto outros fizeram avançar o conhecimento da atividade dos avaliadores nestas situações, o que permite tirar algumas lições. Outros estudaram, ainda, os efeitos positivos, mas também perversos dessas práticas. Em suma, esta revisão de literatura poderá interessar aos profissionais, aos docentes e aos formadores profissionais que queiram conhecer os avanços na investigação sobre estas questões.

Note de l'auteure: La correspondance liée à cet article peut être adressée à Claire Tourmen, maître de conférences, Agrosup Dijon, 26, boul. du Dr Petitjean, 21000, Dijon, France, [claire.tourmen@educagri.fr].

L'auteure tient à remercier Sylvie Deblay d'Eduter, qui a contribué à ce travail sur l'évaluation des compétences, et Christophe Dierendonck, qui l'a soutenue dans sa publication. 
Les recherches sur l'évaluation en éducation se sont longtemps centrées sur l'évaluation des apprentissages, à partir notamment des travaux en docimologie ou en psychométrie. Un nouvel objet est apparu depuis une dizaine d'années: l'évaluation des compétences. Les chercheurs ont été interpellés par des praticiens chargés de mettre en place de nouvelles formes d'évaluation, que ce soit la validation des acquis de l'expérience (VAE, en France depuis la loi de 2002) ou des formes d'évaluation centrées sur les compétences dans le cadre de l'approche par compétences (APC, à l'échelle internationale) et d'un mouvement plus global de professionnalisation de l'enseignement supérieur. Ainsi, l'évaluation des compétences constitue un objet nouveau dans le champ de l'évaluation, la notion de compétence étant d'introduction plutôt récente dans le champ des sciences de l'éducation (De Ketele, 2001; Zarifian, 2001).

Ce nouvel objet - les compétences - pose toutefois bien des questions et soulève de nombreux débats. Crahay soulignait la confusion conceptuelle qui entoure cette «caverne d'Ali Baba conceptuelle» dans un certain «vide théorique» (Crahay, 2006, p. 101). Si les débats persistent, il nous semble voir émerger, aujourd'hui, un faisceau de travaux en éducation, en évaluation, en psychologie du travail et en didactique professionnelle qui se retrouvent autour d'une définition des compétences dont nous allons tenter de dresser le contour. Cette définition, qui lie compétences et situations, a de nombreuses implications pratiques que nous soulignerons.

Nous allons nous baser sur une revue de littérature des écrits en évaluation, en sciences de l'éducation et en psychologie du travail qui ont porté sur le thème de l'évaluation des compétences professionnelles ${ }^{1}$. Ce thème étant au croisement de plusieurs disciplines, il a en effet été traité sous l'angle évaluatif (littérature en évaluation), sous l'angle des compétences au travail (littérature en psychologie du travail et en didactique professionnelle) ou sous l'angle éducatif et développemental (littérature en éducation), ce qui explique les trois entrées retenues. Ainsi, nous traiterons prioritairement de l'évaluation des compétences pour et dans l'enseignement professionnel, qui était notre focale d'entrée et qui donnait une unité à nos lectures, même si nous avons cité quelques études de réfé- 
rence concernant l'enseignement scolaire initial - qui n'est pas abordé de front, car cela aurait nécessité un autre travail équivalent (voir par exemple Crahay, 2014). De même, nous n'avons cité que quelques études portant sur l'évaluation des salariés sur les lieux de travail, ce qui appellerait également, pour être exhaustif, d'autres lectures (Lévy-Leboyer, 2011; Loarer, 2014). Nous n'avons donc pas abordé de front la question de l'évaluation des compétences scolaires ${ }^{2}$ et renvoyons pour ce faire au récent ouvrage de synthèse de Dierendonck, Loarer et Rey (2014). Enfin, nous n'avons pas discuté la notion de compétence dans les autres champs où elle a pu être aussi étudiée: en sociologie (hormis quelques références proches du domaine de l'éducation ou du travail), en sciences du langage et en sciences de gestion. C'est sur les compétences professionnelles et leur évaluation que nous avons centré notre propos.

Ces limites étant posées, nous avons souhaité voir s'il était possible de dégager une synthèse de cette littérature - où en est la recherche, aujourd'hui, sur la question de l'évaluation des compétences professionnelles? - et des enseignements à visée pratique pour les enseignants/formateurs en demeure d'évaluer des compétences professionnelles, sur le terrain, dans la perspective de former de futurs professionnels. Nous avons choisi les textes des principaux auteurs des domaines cités qui traitaient de manière explicite (dans le titre ou dans le résumé) de la question de l'évaluation des compétences professionnelles.

Que nous ont appris plus de vingt années de recherches sur l'évaluation des compétences dans les domaines cités? Loin de viser l'exhaustivité devant l'incroyable quantité de littérature que ce sujet a occasionnée, nous tenterons une synthèse orientée par un souci: chercher des points de convergence au-delà des points de débat et repérer des avancées sur la connaissance des phénomènes en jeu dans les situations d'évaluation des compétences professionnelles. Et des avancées, il y en a eu. Elles passeraient presque inaperçues dans la masse des publications. Notre ambition est de les mettre en avant sous un jour nouveau.

Nous avons tiré de nos lectures une synthèse que nous avons organisée en quatre temps. Devant une pratique comme celle de l'évaluation des compétences professionnelles, les chercheurs ont en effet adopté plusieurs angles d'attaque:

1. Documenter l'émergence de ces pratiques et en discuter la nouveauté; 
2. Tenter de définir les processus en jeu et de proposer des cadres et des modèles pour décrire et/ou prescrire ces pratiques;

3. Observer ces pratiques pour tenter d'en analyser le déroulement, la logique et les risques;

4. Documenter les effets attendus, inattendus, voire pervers de ces pratiques, dans une perspective plus large.

Chacun de ces angles a permis des avancées et ouvert des champs de questionnement et de vifs débats. Nous nous attarderons sur ce qui nous semble une des avancées majeures des dernières années, soit la convergence de nombreuses études qui définissent les compétences professionnelles en lien avec la maîtrise des situations. Nous analyserons les implications pratiques de cet embryon de définition, ses limites ainsi que les risques qu'il soulève, en montrant notamment comment Vergnaud (2001) a pu en proposer un développement fécond.

Nous tenterons de dégager, en conclusion, les principaux enseignements de cette revue de littérature.

\section{Évaluation des compétences: une nouvelle classe de situations?}

\section{La multiplication des situations d'évaluation des compétences}

Un des premiers points discutés dans la littérature est la nouveauté des situations où il est demandé d'évaluer des compétences, dans le monde éducatif comme professionnel. Le développement rapide de ces situations est indéniable depuis les années 1980. En effet, le concept de compétence a émergé dans les années 1980 dans certains univers du travail, et plus particulièrement du patronat (Zarifian, 2001; Crahay, 2006), avant d'être généralisé à l'ensemble des milieux professionnels, dont ceux de la formation professionnelle et de l'éducation.

Dans le cas de l'enseignement professionnel, il est aujourd'hui préconisé d'évaluer des compétences à différents moments, selon différentes modalités, avec différents outils et pour différentes finalités (formatives, sommatives, recrutement et positionnement, etc.). Dans certains cas, les compétences sont évaluées par les enseignants, dans d'autres par les tuteurs de stage, dans d'autres cas encore par des jurys mixtes. Comme le remarquent Tardif et Dubois (2010), l'évaluation des compétences en formation 
peut ressortir d'une «logique photographique» (estimation d'un niveau à un moment $\mathrm{M}$ ) ou d'une «logique vidéographique» en prenant en compte une progression sur un temps long. Portfolios et carnets de compétences sont ici utilisés, comme en témoigne leur succès récent et fulgurant (Lacourse \& Oubenaïssa-Giardina, 2009), ou bien encore des dossiers spécifiques qui témoignent des activités passées (un dossier de VAE, par exemple). Les injonctions à évaluer des compétences se sont également rapidement développées dans le monde éducatif, comme en témoigne le travail de recension complet de Crahay (2006).

\section{Une nouveauté?}

Ces situations sont-elles pour autant nouvelles et appellent-elles le développement de nouvelles recherches? De nombreux chercheurs affirment que l'évaluation des compétences constitue un objet nouveau, voire une problématique nouvelle dans le champ de l'évaluation des apprentissages. De Ketele (2001) distingue ainsi les évaluations classiques «centrées sur les contenus d'apprentissage» des «savoirs» des évaluations «centrées sur une activité intégratrice», qu'il qualifie d'assez fréquentes dans les réseaux d'Éducation nouvelle. «Ces pratiques consistent à choisir une activité susceptible de mettre en jeu les contenus et/ou activités et/ou thèmes les plus importants ayant fait l'objet des apprentissages antérieurs » (De Ketele, 2001, p. 39).

De plus, de nombreux chercheurs se sont emparés de cette notion, récente dans le champ des sciences de l'éducation comme de la psychologie du travail. Il s'agirait d'un objet nouveau pour l'évaluation et pour la recherche en évaluation et en éducation.

D'autres auteurs en éducation, comme Perrenoud, rejettent toutefois la complète nouveauté de la question de l'évaluation des compétences. Selon lui, l'école le fait déjà depuis longtemps et l'évaluation des connaissances n'est pas aussi indépendante de celle des compétences qu'on pourrait le croire. «L'évaluation des compétences n'est pas un problème neuf, elle oblige à remettre sur le métier la question de l'évaluation des connaissances et à voir que ces problématiques sont plus proches qu'on ne croit» (Perrenoud, 2004, p. 8).

Ce qui semble finalement nouveau, c'est la prégnance du concept de compétence - qui a envahi les univers du travail et de la formation depuis les années 1980 - et les pratiques que cela encourage ou remet en ques- 
tion, même si, auparavant, des compétences étaient déjà évaluées, tout comme monsieur Jourdain faisait de la prose sans le savoir (Perrenoud, 2004). Ce qui est nouveau, donc, c'est l'injonction à développer et à professionnaliser des formes d'évaluation des compétences dans les univers du travail et de la formation. Nous sommes devant un «concept qui séduit et qui polarise», selon Serres (2014), et dont l'usage peut même être contesté - voir par exemple Crahay (2014), qui propose de se débarrasser du concept de compétence pour revenir à celui «d'intelligence» tel qu'il a été développé par Piaget (1947). Nous reviendrons sur les nombreux débats que l'émergence de ce concept a suscités.

\section{Les compétences, objets de lutte et de transaction sociale}

Les compétences ne sont pas uniquement un objet de lutte et de débat sur le plan scientifique. Elles le sont aussi sur le plan pratique. Selon des sociologues du travail comme Dujarier (2006), l'objet «compétences» est en effet devenu depuis les années 1990 un objet de transaction sociale de plus en plus valorisé dans les rapports sociaux de travail et de formation. Le recours de plus en plus fréquent au concept de compétence dans les systèmes éducatifs et de formation s'explique par la nécessité croissante de justifier la professionnalisation des élèves, dans le cadre d'une injonction nationale et internationale. Si l'on se rapporte à l'étymologie et à l'origine du terme dans le droit latin («juste rapport»), la compétence est avant tout un adjectif qui qualifie une organisation ou une personne de «compétente pour», qualification qui lui confère le pouvoir légitime de faire quelque chose (un tribunal sera compétent pour juger certains délits, par exemple). Par extension, on en a fait un nom, une qualité: la compétence d'un tribunal, c'est le droit du juge de juger quelque chose ou quelqu'un et, par extension, son aptitude à effectuer certains actes. C'est donc une habileté reconnue qui donne droit à, et qui est l'objet d'une attribution et d'une désignation sociales. La compétence est donc, dès l'origine, un objet de lutte et de distorsion; c'est un concept évaluatif. Si l'on se réfère à des auteurs de psychologie culturelle, on pourrait dire que l'on passe notre temps à déterminer qui est compétent ou non à des fins de classement et d'orientation des personnes, à définir des rôles sociaux, et à distribuer le droit et le pouvoir de les occuper (Valsiner, 2007). La sociologue du travail Dujarier (2006) observe qu'aujourd'hui on vend moins du temps que des compétences. D'où les questions de sa mesure et de construction sociale de la mesure, et de son acceptation par les parties en présence. La volonté est 
alors de pacifier les rapports sociaux par l'objectivation de la mesure lors d'une évaluation. L'évaluation des compétences est donc une situation à enjeux pour les évalués (élèves, salariés) comme pour les évaluateurs (encadrants, formateurs, enseignants). Et les chercheurs s'en sont emparés.

\section{Cadres proposés pour évaluer des compétences professionnelles}

La majorité de la littérature sur l'évaluation des compétences propose des définitions et des modélisations des processus en jeu, à visée descriptive, mais aussi souvent prescriptive. La principale entrée est définitionnelle: comment décrire et définir cet énigmatique objet? Et, par ricochet, comment l'évaluer?

\section{Un champ de bataille}

D'entrée de jeu, soulignons l'existence de multiples définitions des compétences dans les champs de la formation et des sciences humaines, définitions en débat, ce qui ne simplifie pas le travail des évaluateurs, qui risquent de bien souvent commencer par une mise au point sur la signification donnée au concept, au risque de se perdre dans des débats sans fin.

L'ingénierie de formation a en effet produit de nombreuses catégorisations pratiques qui découpent le contenu des compétences en éléments distincts (comme le célèbre triptyque savoirs/savoir-faire/savoir-être) aux fondements non explicités (Stroobants, 1994). Dans le même esprit, un modèle triangulaire KAS (qui agrège knowledge, aptitudes et skills) est fréquemment utilisé en Amérique du Nord (Parry, 1997). Ces multiples catégorisations alimentent une «nébuleuse théorique» autour du concept et des controverses sans fin à son sujet (Jonnaert, 2014).

Ces catégorisations ont été souvent critiquées par les chercheurs pour leurs fondements flous et éloignés de la réalité des phénomènes en jeu dans les univers de travail, pour leur manque de praticité et pour leurs conséquences néfastes. Outre le fait qu'elles découpent la réalité en catégories artificielles et distinctes dont les liens sont ensuite perdus ou problématiques (Mayen, 2001), elles sous-entendent que les personnes seraient les «propriétaires» de leurs compétences (Mayen, Métral, \& Tourmen, 2010), ce dont il s'agirait d'évaluer ensuite la possession ou non. Les évaluateurs ne savent pas toujours comment, d'ailleurs. 


\section{Les convergences vers un élément de définition scientifique}

Toutefois, depuis plusieurs années, des rapprochements ont lieu entre chercheurs en psychologie, en éducation et en évaluation. Si des divergences indéniables demeurent, nous sommes frappée par l'émergence et le succès d'un premier noyau de définition, qui, certes embryonnaire, semble rallier les suffrages. Cet embryon de définition consiste à lier les compétences et les situations.

En effet, aujourd'hui, une convergence est observée entre plusieurs auteurs en éducation et en psychologie pour définir les compétences en référence aux situations (de travail, de vie). Cette convergence trouve son origine dans des travaux en psychologie du travail et du développement, dans une tradition piagétienne. Les psychologues du travail ont en effet depuis longtemps montré que la compétence est ce qui permet l'adaptation (Vergnaud, 2001) et que «l'intelligence du travail» (De Montmollin, 1986) réside dans les interstices où l'activité réelle dépasse la tâche prescrite afin de permettre l'adaptation à la variété des situations réelles et à leur complexité (Leplat, 1997), et ce, même si le concept de compétence est d'introduction récente dans ce champ également ${ }^{3}$. En continuité avec ces travaux, être compétent a été défini en didactique professionnelle comme la capacité à traiter une classe de situations professionnelles quel que soit son état (normal/dégradé) et son degré de complexité (Vergnaud, 2001; Mayen, Métral, \& Tourmen, 2010; Métral, Tourmen, \& Mayen, 2014).

Cette idée est également développée par de plus en plus d'auteurs en évaluation et en éducation. Selon Crahay, la compétence implique «la mobilisation à bon escient de ressources multiples pour résoudre des problèmes complexes» (Crahay, 2014, p. 191). De Ketele va dans le même sens: «Le concept de compétence peut se définir comme la capacité d'une personne à mobiliser un ensemble de ressources (cognitives, affectives, gestuelles, relationnelles, etc.) pour réaliser une catégorie de tâches ou résoudre une famille de situations-problèmes » (De Ketele, 2001, p. 42). Il parle aussi de la capacité à «mobiliser un ensemble de savoirs, savoir-faire et savoir-être pour résoudre une famille de situations-problèmes» (Loc. cit.). Une approche convergente existe chez Roegiers (2014) et Rey (2014), qui lient les compétences à la capacité à faire face à des «gammes» de situations ou à des «familles de tâches». Selon ce premier auteur, «pour prouver sa compétence, l'élève doit pouvoir faire face à n'importe quelle situation de cette famille» (Roegiers, 2014, p. 73). Les travaux du groupe 
international du $\mathrm{BIEF}^{4}$ vont également dans ce sens : être compétent, c'est «résoudre des situations complexes» (Gérard, 2005). C'est aussi le cas de certaines approches par compétences développées en Amérique du Nord et testées dans le cadre de missions internationales (Jonnaert, Barrette, Masciotra, \& Yaya, 2006; Jonnaert, 2014). Paquay, Van Nieuwenhoven et Wouters, des auteurs en évaluation s'appuyant eux aussi sur plusieurs études en psychologie de l'expertise (Dreyfus, Dreyfus, \& Athanasiou, 1986), évoquent la même idée: «Le développement de l'expertise consiste moins en une accumulation de ressources (savoirs, savoir-faire et attitudes nouvelles) qu'en une capacité intégrative d'utiliser adéquatement ces ressources et de façon le plus souvent intuitive face à des situations complexes» (Paquay Van Nieuwenhoven, \& Wouters, 2010, p. 12). Il ne s'agit pas uniquement d'une «extension du répertoire d'activités», mais plus largement «d'une capacité à conceptualiser les situations en fonction des caractéristiques du contexte» (Loc. cit.).

Cette approche a également été développée par Le Boterf (2006) en ingénierie de formation. Selon lui, les compétences sont constituées de «combinaisons de ressources» utilisées pour «gérer un ensemble de situations professionnelles». Toute compétence est donc inséparable des situations, ce qui donne au concept une dimension à la fois «individuelle» et «collective». Cela a pu amener certains chercheurs (Crahay, 2006; Rey, 1996) à rejeter les énigmatiques « compétences métadisciplinaires» revendiquées dans les années 1970 ou les compétences «transversales», où tout lien avec une situation concrète avait été perdu.

Les psychologues du travail Loarer et Pignault soulignent les avantages d'une telle définition: «Les conditions et contextes de l'activité font partie intégrante de la compétence. Ne pas les prendre en compte peut donner l'illusion d'une généralité, voire d'une transversalité accrue de la compétence. Alors que de nombreux auteurs soulignent le caractère spécifique de la compétence» (Loarer \& Pignault, 2010, p. 216). De ces travaux ressort donc un relatif consensus autour de l'idée que les compétences peuvent être définies comme autant de ressources (quelle que soit la dénomination donnée; nous y reviendrons) qui permettent l'adaptation des personnes à des situations de travail variées et variables. Ceci insiste de plus sur le fait que les compétences sont distribuées, qu'elles dépendent aussi des conditions de réalisation de l'activité, et non uniquement des personnes (Mayen, 2005; Mayen, Métral, \& Tourmen, 2010). 


\section{Les conséquences pratiques de cet embryon de définition}

Les auteurs qui lient compétences et situations en tirent une conséquence majeure: pour évaluer des compétences professionnelles, il y a nécessité de passer, d'une façon ou d'une autre, par l'activité de travail en situation. Ce serait un détour nécessaire pour ne pas dériver sur l'évaluation des personnes, comme le souligne Dejours $(2003)^{5}$, mais bien rester sur l'évaluation du travail.

Ce qui pose la question suivante: comment réintroduire les situations professionnelles dans l'évaluation? Tardif et Dubois développent cette idée: si évaluer des compétences correspond à évaluer un «savoir agir», alors elles ne peuvent pas être évaluées «en dehors des situations exigeant une mise en acte» (Tardif \& Dubois, 2010, p. 141). «Cette nécessaire prise en compte des situations est cruciale dans l'évaluation de toute compétence» (Ibid., p. 142). La voie royale pour estimer un niveau de compétences consiste donc à donner à vivre une ou plusieurs situations et à voir si (et comment) la personne s'en sort (tests, épreuves pratiques, etc.). Quand l'observation d'une personne en activité n'est pas possible, les évaluateurs passent par une observation indirecte de l'activité, sur base de ses traces écrites et/ou orales (comme dans un portefeuille de compétences, un dossier de VAE, un entretien de bilan de compétences, etc.). Loarer décrit lui aussi, dans un tableau de synthèse, l'existence «d'approches directes» (sur base d'observations) et «indirectes» (sur base de déclarations) de l'évaluation des compétences (Loarer, 2014, p. 211).

Le succès du lien entre compétences et situations a ainsi donné lieu à toute une ingénierie des situations:

- Comment décrire les situations de travail ou de vie de manière à construire d'authentiques situations d'évaluation? Des «référentiels de situations» ont été construits pour ce faire (Jonnaert et al., 2006; Mayen, Métral, \& Tourmen, 2010);

- Quelles situations choisir? Tout un courant a préconisé l'usage de situations «inédites» et «complexes» (Roegiers, 2001; Le Boterf, 2006) pour révéler la compétence scolaire. Cela ne va pas sans difficulté. Si, selon Rey (2014), évaluer des compétences implique d'identifier des «familles de tâches» dans lesquelles une connaissance ou une procédure seront utiles, comment s'assurer que l'évalué sera capable de faire le «transfert» à une tâche nouvelle, inédite, complexe? En effet, évaluer dans plusieurs situations n'est 
souvent pas possible. Certains auteurs ont contesté la suprématie du critère de la nouveauté - qui risquerait de trop mettre en échec les élèves - au profit de situations plus routinières, mais centrales dans une discipline (Crahay, 2006). Dans l'enseignement professionnel, il a été préconisé de se centrer sur des situations «critiques» (Mayen, 2001), au sens de situations de travail importantes pour la réussite de l'activité, difficiles à maîtriser et centrales pour l'identité d'un métier donné.

\section{Les difficultés pratiques}

Toutefois, le concept de situation est loin d'être stabilisé et clairement défini. Certains soulignent que l'identification de familles de tâches ne va pas sans soulever la question de la singularité même des problèmes éprouvés, ce qui met en cause la fiabilité de tout le processus d'évaluation (Rey, 1996). La réponse apportée à cette question par les travaux en didactique professionnelle repose sur l'unité théorique inséparable entre les situations et les concepts. Selon la théorie des champs conceptuels développée par Vergnaud (1990) et inspirée des travaux de Piaget (1947), une «classe de situations »- ou de problèmes - se caractérise par des «concepts»-et les «schèmes» associés - qui permettent de la traiter. Évaluer la maîtrise de certains concepts passerait donc par l'évaluation de la maîtrise de certaines situations d'une même classe, et inversement.

L'appel à évaluer les compétences par la mise en activité a également soulevé des problèmes de faisabilité dans le système d'enseignement traditionnel. L'organisation d'épreuves «situées» semble difficile dans un cadre scolaire, et même dans le cadre de la formation professionnelle, où le format scolaire reste prégnant (Métral, Tourmen, \& Mayen, 2014; Tourmen, Deblay, Lipp, \& Boude, 2013). Selon le chercheur en éducation Perrenoud, «l'école n'est pas l'endroit idéal pour créer de vraies situations de raisonnement et d'action» (Perrenoud, 2004, p. 8), car les enseignants sont attirés par la facilité du test de connaissances («Qui a inventé l'imprimerie?») qui permet une correction rapide, standardisée, «donc équitable et irréprochable». À l'inverse, «sortir du papier crayon» et donner à vivre des tâches moins abstraites aux élèves, tour à tour, prend du temps, soulève le risque de l'inéquité («puisque tous les élèves ne sont pas confrontés à la même tâche») et rend l'évaluation plus complexe, moins standardisée. Perrenoud (2004) souligne que ceci n'est pas toujours évident pour un enseignant, car c'est «en rupture avec son mode habituel d'enseigne- 
ment» et cela nécessite de penser l'apprentissage en termes de situations («situations d'apprentissage» et «situations d'évaluation», qui ne sont pas antinomiques) et d'activités, avant de penser aux savoirs et aux exercices traditionnels.

\section{La boîte noire}

Reste une inconnue, une boîte noire: qu'est-ce qui fait le lien entre les compétences et les situations? À quoi l'évaluateur doit-il être attentif dans l'activité des personnes en situation? Les auteurs cités précédemment parlent, pour en rester à un consensus mou, de «ressources». La neutralité de ce terme est appréciable, mais masque un flou conceptuel évident. Ce qu'entendent ces auteurs par ce terme diverge: des «attitudes», des «savoir-faire» ou des «savoirs» (parfois qualifiés de «procéduraux»). On retombe dans une multitude floue de modèles et de concepts, dans ce «vide théorique» dont parlait Crahay (2006). Et l'évaluation des compétences peut, sous couvert de ce vide, être rabattue sur les anciennes modalités d'évaluation des savoirs scolaires.

Cette difficulté est renforcée par l'objet lui-même, qui se dérobe sans cesse à l'observation. En effet, les évaluateurs ne peuvent pas «voir» les compétences. L'objet doit être provoqué, mis à jour, inféré. Selon le psychologue du travail Dejours, «l'essentiel, en effet, de ce que l'on cherche à évaluer se dérobe à l'observation directe» (Dejours, 2003, p. 12). Loarer (2014) affirme également que la compétence ne se donne jamais à voir directement.

Ceci laisse la porte ouverte à toutes les catégorisations possibles et ouvre un difficile chapitre de la psychologie: qu'est-ce que faire, savoir faire, connaître, réussir, apprendre? Rien de moins. L'évaluation des compétences débouche sur des questions redoutables de recherche fondamentale.

\section{La nécessité d'une théorie de l'activité intelligente et de son développement}

Pour sortir de la confusion, certains auteurs ont recours à des travaux de psychologie de l'expertise, du développement et du travail. Plusieurs théories du développement de l'activité intelligente existent en effet et peuvent être prises pour point de départ (par ex., Piaget, 1947; Vergnaud, 2001 ; Anderson, 1982; Dreyfus, Dreyfus, \& Athanasiou, 1986; Engeström \& Sannino, 2010), comme le soulignait Crahay (2006). Elles 
n'auront pas les mêmes conséquences méthodologiques en évaluation, comme nous allons le voir en développant l'exemple de la théorie des schèmes de Vergnaud $(1990,2001)$ et ses conséquences en évaluation des compétences. Nous avons choisi cette théorie, car nous sommes persuadée qu'elle peut offrir un cadre théorique fécond et que son usage en évaluation, développé par Vergnaud dans un article de 2001, est trop souvent resté inaperçu, sauf dans quelques travaux de didactique professionnelle (Métral, Tourmen, \& Mayen, 2014).

\section{La théorie des schèmes et ses conséquences pratiques en évaluation}

Sans revenir en détail sur la théorie des schèmes développée par Vergnaud (1990, 2001) dans la lignée des travaux de Piaget (1947) sur le développement de l'intelligence chez les enfants, nous repartirons de l'idée que les compétences sont ce qui favorise l'adaptation aux situations, et que «ce qui s'adapte, ce sont des schèmes» (Vergnaud, 2001), ou des «structures internes d'action qui peuvent être activement répétées» (Piaget, 1947, p. 13) organisées par des «invariants opératoires», soit des «règles d'action», «théorèmes en actes» et «concepts en actes» reliés à des «buts» (Vergnaud, 2001). Or, ces ressources sont en partie cognitives, partiellement conscientes pour les individus eux-mêmes et, donc, difficilement observables.

Une des premières conséquences en évaluation est que ce qui organise l'activité compétente se voit mal. En cause, un phénomène bien connu des ergonomes et des psychologues du travail est rappelé par Vergnaud: «Le décalage entre les connaissances mises en œuvre dans l'action effective et celles qui sont aisément explicitées concerne tous les registres et tous les niveaux de compétences. Comme dans un iceberg, la partie immergée de nos connaissances est considérable» (Vergnaud, 2001, p. 45). Le théoricien est en cela cohérent avec les observations faites par d'autres auteurs en psychologie du travail. «Tous ces savoirs nés de la pratique du travail, parce qu'ils sont pour une bonne part clandestins, sont souvent mal relayés par le langage. Les mots pour désigner, décrire, caractériser ces savoir-faire sont chroniquement déficitaires. Du fait de cette difficulté à "sémiotiser"une partie importante des savoir-faire, l'évaluation du travail a toutes les chances d'être lacunaire» (Dejours, 2003, p. 19). Ainsi, «l'intelligence professionnelle, en règle, est en avance sur sa connaissance et sa symbolisation» (Ibid., p. 22). «Cette connaissance "par corps" n'est 
pas [...] l'affaire des travailleurs manuels seulement. Les agents eux-mêmes sont en grande difficulté pour formaliser leur expérience et, par voie de conséquence, leurs compétences qu'ils méconnaissent» (Ibid., p. 36). Baillé rappelait aussi que «l'expert n'est pas nécessairement expert de sa propre expertise», notamment des «savoir-faire locaux intelligents» (Baillé, 2001, p. 63) qui constituent des schèmes non formalisés et appartiennent à ce que Leplat (1997) appelle «la compétence incorporée».

Conséquence logique, l'autoévaluation demandée aux individus peut être très limitée et fortement biaisée. Plusieurs études ont ainsi montré les limites de l'autoévaluation: on peut facilement se méprendre sur son niveau de compétences. En cause, un phénomène de distorsion de la réalité largement étudié en psychologie sociale ${ }^{6}$. Citons par exemple l'effet Dunning-Kruger (datant de 1999), selon lequel les personnes les plus compétentes ont tendance à se sous-estimer, et inversement. Les psychologues du travail s'accordent pour dire qu' «il est très difficile pour une personne de mettre en mots son expérience» (Loarer \& Pignault, 2010, p. 213), d'autant plus pour les niveaux faiblement qualifiés. Mais pas seulement pour ces niveaux: les mots manquent pour parler de son expérience. En tant que fruits/produits de l'expérience, les compétences sont un construit social qui n'est pas complètement transparent à nous-mêmes et qui nécessite une élaboration (Mayen, 2009) 7 .

Une autre conséquence directe sur laquelle insiste Vergnaud est qu'il faut passer par l'analyse de l'activité en situation, comme nous l'avons déjà évoqué, et ce, quelles que soient les méthodes utilisées.

\section{Les risques de l'évaluation des compétences}

Observer l'activité (en cours ou passée) comporte toutefois des risques, soulignés par Vergnaud, mais aussi par d'autres auteurs en psychologie du travail:

- Le risque d'en rester à ce qui est observable de l'activité, aux gestes, aux comportements, aux résultats (en se centrant sur les seuls critères de performance ou de conformité, voir Baillé, 2001), dans une définition limitée de l'activité qui confond «activité réelle» et «réel de l'activité» (Clot, 2008) et qui méconnaît donc tout ce qui n'est pas réalisé. Le risque est que les évaluateurs se limitent à quelques indicateurs trompeurs ou réducteurs, comme le souligne aussi le psychologue du travail Dejours: «Le temps de travail ne mesure 
que la durée de l'effort et ne restitue rien de son intensité, ni de sa qualité, ni de son contenu» (Dejours, 2003, p. 10). «Il arrive même souvent que les tâches qui demandent le plus d'effort soient celles dont les résultats matériels sont les moins flatteurs» (Ibid., p. 32). De quel point de vue la performance est-elle envisagée: celui de l'organisation, des clients ou usagers, des gestionnaires, des travailleurs, des métiers? De plus, quel statut est laissé aux inévitables échecs et erreurs du travail réel? «Le réel se fait connaître au sujet par sa résistance aux procédures, aux savoir-faire, à la technique, à la connaissance, c'est-à-dire par la mise en échec de la maîtrise. Travailler, c'est échouer. Le monde réel résiste» (Ibid., p. 14). Et Dejours de souligner les risques d'un «mesurage» uniquement quantitatif du travail.

- Le risque de rester centré sur des critères de conformité de l'activité au regard de procédures attendues, ce qui risquerait d'enfermer l'activité dans une répétition vide de sens. Baillé (2001), comme Dejours (2003), observe que, dans l'activité, on utilise et on transforme les «savoirs institutionnellement reconnus» et les procédures prescrites selon son «style» et des «ficelles». «Une évaluation des compétences devrait s'attacher à décrire les procédés par lesquels les objets concrets ou les règles sont détournés pour devenir des instruments pour l'action» (Baillé, 2001, p. 64), et non se réduire à une simple mesure de conformité avec un rôle écrasant donné à la prescription.

- De même, grand est le risque de négliger les savoirs issus de l'expérience au profit des savoirs disciplinaires, fortement valorisés dans un cadre scolaire. Comme le rappelle Baillé, «ces compétences tacites sont rarement prises en compte dans la qualification, voire dévalorisées eu égard aux connaissances disciplinaires » (Baillé, 2001, p. 63).

\section{La descente vers le cognitif préconisée par Vergnaud}

Afin de limiter ces risques, Vergnaud (2001) souligne l'importance d'évaluer aussi ce qui organise l'activité, à savoir sa dimension cognitive, ce qui implique de disposer d'une palette de critères et d'indicateurs larges et «non mutilants», comme le souligne Baillé (2001). Fondant en cela une perspective de didactique professionnelle sur la question, Vergnaud soutient que «l'évaluation de la compétence appelle non seulement l'analyse du résultat de l'activité, mais aussi celle de son organisation» (Vergnaud, 
2001, p. 47). Il appelle cela «la descente vers le cognitif». «Pas d'analyse des compétences sans analyse de l'activité, pas d'analyse de l'activité sans analyse des conceptualisations sous-jacentes, puisque ce sont elles qui, en dernier ressort, font la différence entre un niveau de compétences et un autre» (Loc. cit.). Il invite donc les évaluateurs à ne pas être contaminés par le béhaviorisme et à étudier la représentation, même si cela est difficile. «Et pourtant il faut avancer!», conclut-il.

Le chercheur en éducation Baillé va dans le même sens: "Une évaluation de l'activité (de ce qui est à l'œuvre pour l'exécution des tâches) devrait rendre compte du passage du cognitif (conceptuel, opératoire) à l'opératif par une construction inversée dont ce dernier constitue le point de départ en raison de son accessibilité» (Baillé, 2001, p. 57). Il conclut que l'évaluateur, «à travers l'expertise de sujets en situation de travail», doit maîtriser les outils et les concepts de l'analyse de l'activité, et notamment savoir analyser les schèmes. "Pour les rendre observables», la première opération consiste à «déterminer la classe de situations dans lesquelles ils sont actifs». La seconde consiste à identifier les «procédures canoniques de résolution» à mettre au regard de celles «effectivement activées par l'élève» (Ibid., p. 62). Perrenoud souligne aussi que:

le fonctionnement intellectuel est dans une large mesure invisible et il ne suffit pas d'observer des faits et gestes pour le reconstituer. L'évaluateur a besoin d'observer l'élève au travail de près, durant un bon moment, dans des phases décisives. Il doit pouvoir accéder à sa pensée, l'interviewer, avancer et confirmer des hypothèses qu'il infère de la conduite observable des manières probables de raisonner (Perrenoud, 2004, p. 10).

L'expertise de l'évaluateur consiste alors à 1) «inventer des situations propices» et à 2) "savoir ce qu'elles mettent en évidence». L'enseignant devient un «observateur professionnel des élèves au travail» avant d'être un «compteur de points» ou un «correcteur de travaux écrits» (Perrenoud, 2004, p. 10).

La nécessité d'évaluer l'organisation de l'activité au regard de sa réalisation a été renforcée par les travaux de Chenu (2012) sur des étudiants en secrétariat et en bureautique. Après l'analyse de la réalisation d'une tâche de traitement de texte suivie d'un entretien d'explicitation avec 31 d'entre eux, Chenu a montré un découplage entre la performance observée et la connaissance des procédures. Ainsi, ce n'est pas parce que certains étudiants connaissaient les procédures à appliquer ou en énonçaient 
certains principes oralement qu'ils obtenaient de meilleurs résultats. De plus, certains étudiants parmi les plus performants énonçaient une multitude de règles d'action non fédérées que l'auteur a appelées des "préoccupations». La triangulation d'indicateurs provenant de la réalisation de l'activité, de ses résultats et de ses organisateurs semble donc utile pour obtenir une évaluation aussi complète que possible du niveau de compétences d'une personne.

\section{Les pratiques existantes en formation}

Cette façon de faire ne semble pas inexistante. Comme l'a constaté Lebel (2009), les évaluateurs s'engagent parfois dans une enquête sur ce qui oriente l'activité (voir aussi les observations de Tourmen, 2012; Métral, Tourmen, \& Mayen, 2014). C'est du moins ce que l'auteure a constaté dans les situations d'«évaluations des compétences en milieu de pratique » (Lebel, 2009) ou sur les lieux de stage. «Le formateur de terrain et le superviseur ont non seulement la possibilité d'effectuer des observations in situ mais ils ont également accès au raisonnement clinique du stagiaire. [...] Cela leur permet de mieux saisir ce qui sous-tend l'action, par les questions que le stagiaire se pose, les hypothèses qu'il formule, les stratégies mises de côté parce qu'elles lui semblaient peu ou prou réalistes ou encore les alternatives envisagées et les vérifications effectuées avant de poser un geste. [...] Pour reconstruire ces scénarios et vérifier la pertinence des raisonnements du stagiaire, il s'avère important de l'interroger pour comprendre comment il réfléchit» (Lebel, 2009, p. 44). Ce qui suppose que l'enseignant «soit en mesure d'interroger le stagiaire et qu'en retour ce dernier sache expliciter sa lecture de la situation» (Loc. cit.). Toutefois, toujours selon Lebel, «cette situation génère certaines peurs chez les évaluateurs car elle donne une place plus apparente à la subjectivité. De fait, il est encore fortement ancré que le caractère objectif de l'évaluation tient aux chiffres additionnés et aux critères observables plutôt qu'au jugement professionnel exercé au regard de la globalité de la situation à analyser » (Loc. cit.).

De son point de vue en didactique professionnelle, Mayen (2005) a aussi observé que certains évaluateurs allaient au-delà de l'estimation d'un niveau actuel et qu'ils tentaient aussi d'estimer «le potentiel de développement» d'un candidat, comme le suggérait Vygotsky (1934/1997). C'est ce que quelqu'un est capable de faire avec l'aide d'un plus expérimenté. Mayen a ainsi observé que les jurys de VAE assuraient souvent une «fonc- 
tion tutorale» envers les candidats, «en complétant les connaissances de l'évalué», en lui «tendant des perches» et en «faisant varier les situations d'évaluation». Il déplore que «dans de nombreuses occasions on se prive de la possibilité d'examiner et de favoriser l'expression du potentiel, de trouver les moyens de relancer ce qui s'exprimerait sans l'aide d'un autre ou sans la présence de certaines conditions» (Mayen, 2005, p. 20).

\section{Ne pas séparer connaissances et compétences}

Une des dernières conséquences du cadre proposé par Vergnaud (2001) est de ne pas séparer connaissances et compétences, comme cela pourrait être fait. En effet, la descente vers le cognitif à partir de l'activité en situation permet de mettre à jour les connaissances - et les représentations limitées ou erronées, si c'est le cas - qui organisent l'activité d'une personne, souvent même sans qu'elle sache les formuler clairement. Cette prise en compte des connaissances au cœur de l'activité va dans le sens que plusieurs auteurs recommandent (voir Crahay, 2006). Selon ce dernier, les compétences et les connaissances ne sont pas antinomiques. Comme l'a aussi constaté Perrenoud, évaluer des compétences amène les enseignants à «renouveler fortement la problématique de l'évaluation des connaissances et se soucier de leur usage en contexte» (Perrenoud, 2004, p. 9). Car l'évaluation des compétences permet, comme nous l'avons vu, de se questionner sur l'usage des connaissances dans l'action et donc, aussi, d'estimer le niveau de connaissances d'un élève et d' « évaluer l'utilisation fonctionnelle de connaissances disciplinaires» (Loc. cit.). C'est le statut de ces connaissances et leur expression qui changent par rapport à des épreuves plus classiques dites «de restitution de connaissances» de type questionnaire à choix multiple (QCM). Le statut du savoir par rapport à l'activité reste toutefois un problème théorique et pratique majeur, complexe et controversé que nous n'avons pas la prétention de résoudre ici.

\section{Activités des évaluateurs quand ils s'intéressent aux compétences}

D'autres recherches ont tenté d'observer, de décrypter et de comprendre ce que font des professionnels (enseignants, formateurs, encadrants professionnels et maîtres de stage) quand ils évaluent des compétences scolaires ou professionnelles. Ceci afin de ne pas partir d'une définition scientifique de la compétence, mais bien des usages spontanés 
qui en sont faits sur les lieux de travail. Ces recherches ont porté sur des enseignants et des jurys de VAE. Elles permettent de mettre à jour plusieurs phénomènes, que nous allons passer en revue, mais que nous ne pourrons détailler, faute de place.

\section{La façon dont les évaluateurs utilisent des outils : les référentiels et les grilles de critères}

Le premier constat porte sur le fait que l'évaluation des compétences est une activité outillée et qu'il est fait usage de référentiels, de grilles de critères, etc. Si ces artefacts sont instrumentés dans l'activité (et parfois construits ad hoc), ils ne résolvent toutefois pas toutes les difficultés éprouvées par les évaluateurs.

Les psychologues du travail Loarer et Pignault soulignent l'intérêt des référentiels de postes, de métiers et de compétences qui servent d'éléments de référence aux évaluateurs (désignant les tâches et situations à maîtriser, fournissant des modèles d'activité et désignant des ressources attendues), mais aussi leurs limites: «Le passage par ces référentiels et nomenclatures conduit donc immanquablement à une imprécision et à un appauvrissement du contenu pris en compte par rapport à la réalité de l'activité professionnelle» (Loarer \& Pignault, 2010, p. 212). Ils sont imprécis et souvent éloignés de la réalité du travail et de ses variations dans les différents emplois. De plus, dans de nombreux domaines professionnels comme les métiers du service ou les secteurs en voie de professionnalisation, il n'existe pas «one best way» ou une seule bonne manière de réaliser l'activité, ce qui complique la tâche des évaluateurs et fait appel à leur expertise professionnelle.

De plus, si les évaluateurs utilisent les référentiels à leur disposition, les observations montrent qu'ils recourent aussi à d'autres référents (voir par exemple les travaux de didactique professionnelle sur les jurys de VAE de Mayen, Mayeux, \& Savoyant 2006), leurs propres modèles de l'activité et des compétences, souvent implicites (Tourmen et al., 2013). Ces modèles sont liés à leur connaissance «intime et en acte» du métier (Mayen, Métral, $\&$ Tourmen, 2010) et leur permettent de constituer un corps de référence plus ajusté à la réalité du travail demandé. L'usage des référentiels institués amène ainsi toujours à leur réélaboration par les évaluateurs, au regard d'éléments de référence plus personnels et contextualisés. On peut qualifier ce travail de «référentialisation» en acte tout au long de l'évaluation (Figari, 1994), où les évaluateurs reconstruisent sans cesse la référence eu 
égard à une situation donnée. Comme le soulignait Mayen, «si l'on veut évaluer les compétences, il faut déjà savoir quel type de performance et quel niveau de performance on attend de la personne» (Mayen, 2005, p. 23). Attend-on des résultats à court ou à long terme? Sur une tâche répétitive ou à forte variabilité? Sur une situation de quel niveau de complexité? L'auteur conclut que «se mettre d'accord sur les niveaux de performance attendus, c'est à [s]on avis une des premières tâches de ceux qui ont à évaluer des compétences» (Loc. cit.). Ceci peut être une construction collective en cas de coévaluation.

De même, la constitution de grilles de critères et de barèmes de notation est une garantie d'équité, mais elle ne fait pas tout. Comme l'ont montré des études dans un contexte scolaire, concernant la notation des copies du baccalauréat, "l'utilisation d'un barème de notation au point près, voire au demi-point près, ne constitue pas une garantie de précision de la correction » (Merle, 2007, p. 112). Tout dépend de la manière dont les évaluateurs vont interpréter les critères et de leur degré d'exigence: «La finesse d'un barème ne permet la précision de la notation que s'il existe une définition précise, entre les différents correcteurs, sur ce qui est attendu exactement pour telle ou telle question, que celle-ci soit notée sur 3 points ou sur un seul» (Ibid., p. 113). La solution résiderait-elle dans la discussion entre évaluateurs, comme le suggère Merle? «Il ne faut pas attendre une grande efficacité de l'introduction d'un barème dans la correction d'une dissertation en dehors d'une socialisation longue des correcteurs à des exigences effectivement communes. La discussion sur un barème constitue évidemment une des modalités de cette socialisation effective» (Loc. cit.). Le même phénomène est-il agissant dans l'évaluation des compétences en formation professionnelle?

\section{Les évaluateurs recourent à une variété d'indicateurs de compétences}

Un second phénomène a été mis à jour: pour porter leurs jugements, les évaluateurs «triangulent» différentes données (Allal \& Mottier Lopez, 2009). En effet, devant la difficulté d'estimer un niveau de compétences, les évaluateurs s'en remettent souvent à une multiplicité d'indicateurs portant sur différentes dimensions de l'activité et prélevés par différents moyens. Plusieurs recherches sur les pratiques d'évaluation ont mis à jour un phénomène de triangulation des données comme moyen de validation et d'élargissement de la compréhension des phénomènes (Allal \& Mottier 
Lopez, 2009), notamment dans le cas d'élèves dont le niveau est difficile à estimer clairement. «Une triangulation élargie semble donc nécessaire surtout dans des situations d'incertitude, lorsque l'enseignant hésite entre deux notes et ressent le besoin de composer un portrait aussi complet que possible des compétences de l'élève» (Allal \& Mottier Lopez, 2009, p. 37). Les enseignants utilisent de fait une multitude de sources de recueil d'information, en allant des épreuves instituées aux travaux réalisés en classe, aux interactions avec l'élève et ses parents, et même avec d'autres enseignants ou membres du personnel de l'établissement, et à la connaissance du parcours de l'élève et de sa situation familiale.

\section{L’identification des critères et des indicateurs de compétences utilisés}

Ainsi, les évaluateurs appuient leurs jugements sur des critères et des indicateurs multiples. Plusieurs auteurs ont proposé de catégoriser les critères et les indicateurs de compétences utilisés en référence à des théories psychologiques de l'activité (Mayen, 2005; Tourmen, Mayen, \& Figari, 2009; Mayen \& Vanhulle, 2010). Mayen (2005) propose de classer ainsi les critères et les indicateurs de compétences qui peuvent être utilisés: les évaluateurs s'intéressent 1) «aux buts», 2) aux «indices de situation» prélevés par la personne ou aux «prises d'information», 3) à «l'utilisation de règles d'action», 4) à «la connaissance de certains gestes», et 5) à «la conceptualisation» qui fonde et soutient l'action, y compris chez les bas niveaux de qualification. L'auteur note que «la compétence est difficile à mesurer» et qu'il «reste évidemment une zone d'incertitude. C'est toujours une sorte de pari» (Mayen, 2005, p. 29). En effet les évaluateurs fonctionnent par sondage sur une partie de l'activité pour généraliser leurs jugements à la totalité de l'activité. Mayen ajoute: «Je ne suis pas sûr que les autres formes d'évaluation ne relèvent pas du même pari» (Loc. cit.).

Dans un texte ultérieur de Mayen et Vanhulle (2010), ils discutent l'usage de trois «repères du développement». Tout d'abord, l'observation d'une performance permet d'observer 1) «une extension des répertoires d'activité», une «meilleure résistance aux perturbations», des modes d'action «plus économiques» et plus «sûrs» pour la santé. Ensuite, l'observation ou l'inférence de l'organisation de l'action permet d'observer 2) la prise en compte de buts différents, la référence à des lois du travail, l'usage de certains modes de raisonnement et de certaines connaissances, etc. Enfin, on peut observer 3) «la construction d'une position professionnelle» 
dans un environnement collectif ou la «redéfinition des statuts, place et rôle» dans un rapport aux autres qui peut changer la nature du travail. Tout ceci amène à évaluer le développement de nouvelles «manières de prendre la situation et d'agir avec elle», et, éventuellement et dans un second temps, à «se détacher du concret de la situation et de moins en être le jouet» (Mayen \& Vanhulle, 2010, p. 233). Les auteurs insistent toutefois sur le fait que ces repères n'existent pas dans l'absolu, mais sont nécessairement relatifs à des situations professionnelles et d'apprentissage précises et spécifiques.

Enfin, Tourmen, Mayen et Figari (2009) ont distingué, chez les jurys de VAE, des critères portant sur 1) les situations vécues (adéquation au diplôme ou métier visé, variété, complexité, etc.), 2) les activités réalisées (réussite, autonomie, richesse, niveau, etc.), et 3) les connaissances et capacités acquises (usage de termes techniques, capacité de recul, etc.). Ce même modèle s'est révélé pertinent pour analyser les critères prélevés par des enseignants lors d'épreuves d'évaluation en situation professionnelle (Tourmen, 2012), où des critères portant sur ces trois dimensions sont renseignés et mis en balance dans la production des jugements finaux. Des circonstances liées à la difficulté des situations vécues peuvent par exemple contrebalancer une activité à la performance moyenne, ou l'exploration des connaissances sous-jacentes peut venir révéler que l'activité est plus hasardeuse et imitative - et donc moins intelligente - qu'il n'y paraît (Tourmen et al., 2013).

\section{Les évaluateurs construisent leurs jugements de manière dynamique et plus ou moins contrôlée}

Un troisième phénomène a été mis à jour: les évaluateurs sont dans une démarche d'enquête et l'on peut observer que leurs jugements sont le fruit d'une construction dynamique qui commence dès les premiers moments de l'évaluation et qui continue tout au long du processus (Merle, 2007; Tourmen, 2009). Ils font des allers et retours entre des premiers jugements, les données issues de l'observation et les références convoquées (Tourmen et al., 2013). En cas d'évaluateurs multiples, les moments de délibération sont aussi des moments d'arbitrage, comme dans le contexte des épreuves du baccalauréat: "Le sens des délibérations est de surmonter les évaluations divergentes et d'élaborer un modus vivendi qui satisfait les différentes parties prenantes» (Merle, 2007, p. 164). 
Les auteurs posent finalement la question du contrôle des biais dans le processus de jugement. Comme dans toute évaluation se pose la question de fiabilité de la mesure et de son équité. La question de l'équité se pose d'autant plus que l'épreuve d'évaluation des compétences est moins facilement standardisée (Perrenoud, 2004). À trop vouloir «rendre les notes indépendantes du jugement du professeur», le risque est de tomber dans «le questionnaire à choix multiple dépouillé par ordinateur», comme «modèle de l'objectivité» (Perrenoud, 2004, p. 8). À l'inverse, Perrenoud appelle à accepter le fait que l'évaluation est «une tâche complexe» et à restaurer la confiance dans l'expertise de l'enseignant évaluateur à deux conditions: 1) que l'organisation garantisse l'expertise et l'éthique de l'évaluateur, et 2) que le système mais aussi les enseignants ne favorisent pas la logique sélective et sommative au détriment de la logique formative, faisant de l'évaluation une «menace» pour les élèves et leurs parents, puis encourageant par là même des stratégies de défense et d'évitement de leur part. De plus, plus l'enjeu est grand, plus le jugement doit être «justifié», c'està-dire «fondé sur des arguments», même si, «en temps normal», c'est la «confiance» en l'évaluateur qui doit l'emporter (Ibid., p. 11).

La littérature sur l'évaluation des apprentissages en milieu scolaire permettant de bien documenter la question des biais (biais de confirmation, effet d'attente, poids des premiers jugements, etc.), nous n'y reviendrons pas (voir notamment Merle, 2007). Certains biais semblent toutefois propres à l'évaluation des compétences, par exemple celui de «naturaliser les compétences» de certains acteurs en fonction de leur sexe, comme le fait d'attribuer des qualités «innées» aux femmes ou de leur en refuser d'autres (Dejours, 2003). Les recherches en édumétrie (Cardinet, 2003) insistent également sur les biais propres à l'opération d'inférer un trait latent (une compétence) à partir d'une seule situation, lorsque ceci est le cas, comme lors d'une épreuve pratique unique. Il existe en effet des biais liés à la construction des épreuves («Questions», «Moment», voir Cardinet, 2003).

De plus, comme l'ont observé plusieurs auteurs, les évaluateurs ne sont pas tous conscients de ces biais et tous n'en font pas un objet conscient, dans une démarche épistémologique volontaire de prévention des biais (Tourmen \& Mayen, 2012). Merle observe ainsi que «faire part de sa "cuisine" évaluative et partager ses doutes nécessitent de dévoiler les limites de son propre jugement. Cette mise en cause professionnelle ne constitue pas une démarche ordinaire» (Merle, 2007, p. 61). D'autres auteurs pré- 
conisent aux évaluateurs de s'initier à une véritable gestion des biais dans leur processus de construction des jugements (Allal \& Mottier Lopez, 2009). Différentes méthodes d'évaluation des salariés au travail présentent également différentes "qualités métriques» dont les décideurs sont loin d'être toujours au fait, comme l'explique Lévy-Leboyer (2011) dans un ouvrage détaillé sur ce sujet.

\section{Effets souhaités et effets pervers de l'évaluation des compétences}

\section{Les effets d'apprentissage et de motivation}

Des recherches en éducation, en psychologie et en sociologie portent enfin plus spécifiquement sur les effets de ces pratiques. Parmi les effets possibles et attendus de l'évaluation des compétences professionnelles, citons tout d'abord un effet sur l'apprentissage et le développement des personnes évaluées. Comme le rappellent Paquay, Van Nieuwenhoven et Wouters, «a priori, on peut faire l'hypothèse que les évaluations, qu'elles soient ou non institutionnelles, dans leur fonction de feed-back informatif, offrent aux individus et aux équipes des indications susceptibles de les aider à réguler leurs actions en vue d'une efficacité accrue vers les objectifs qu'ils poursuivent et par là même d'apprendre» (Paquay et al., 2010, p. 17). L'évaluation sert ici une fonction de «clarification des systèmes de valeur» en jeu (Jorro, 2010). Mais le «jugement» impliqué dans l'évaluation peut aussi, à l'inverse, provoquer «tensions» et «résistances», jusqu'à venir contrarier, voire pervertir un processus de développement pourtant souhaité (Perrenoud, 2010). L'évaluation peut ainsi stimuler ou bloquer les processus de développement, dans une fonction paradoxale d'accompagnement, mais aussi de sélection/sanction par le contrôle.

Par ailleurs, l'évaluation peut avoir des effets forts sur la motivation et l'implication des élèves. Un effet sur l'engagement dans l'activité a été documenté par le sociologue Pierre Merle: «La notation n'est pas seulement la mesure d'une compétence. Elle influe aussi sur l'investissement dans le travail scolaire via la motivation ou la démotivation qu'elle produit» (Merle, 2007, p. 19). Des études en milieu scolaire ont ainsi montré qu'une notation sévère dès le premier trimestre produit un effet de découragement. À l'inverse, de nombreuses recherches ont prouvé l'existence d'un « effet Pygmalion» où, pour reprendre une idée de Vygotsky (1934/1997), 
la reconnaissance précède la compétence. Le psychologue Dejours a observé le même phénomène à l'œuvre dans les univers du travail: «Travailler, c'est inévitablement souffrir d'une part, prendre des risques d'autre part. La mobilisation subjective [...] repose sur un couple contribution-rétribution. [...] Contrairement à ce que l'on pense communément, la composante de la rétribution qui compte le plus n'est pas sa dimension matérielle (salaire, prime, avancement, etc.), mais sa dimension symbolique» (Dejours, 2003, p. 52). L'évaluation peut donc avoir un réel impact, en retour, sur les relations de travail ou d'enseignement entre un évalué et un évaluateur, ce qui influe sur la situation d'évaluation, car, comme le constate Merle, «le professeur n'évalue pas seulement des compétences, il doit aussi préserver des relations pédagogiques» (Merle, 2007, p. 8).

\section{Les attentes, les craintes et les risques de déception}

C'est donc une situation à enjeux. Toucher aux compétences, c'est entrer sur le territoire de l'autre, son activité, son travail. C'est potentiellement une source de conflits, de stress, de tensions et de négociations (Dejours, 2003; Lévy-Leboyer, 2011), d'autant plus que les personnes sont dans une attitude ambivalente envers l'évaluation de leurs compétences, entre attente de reconnaissance et crainte de sanction. En effet, afin de ne pas être perdants dans les univers du travail et de la certification, les individus semblent être de plus en plus demandeurs d'une aide à l'explicitation et à l'élaboration de leurs compétences, que ce soit dans le cadre de l'accompagnement à une démarche de VAE, pour se préparer à une recherche d'emploi, pour élaborer un portefeuille de compétences et/ou pour faire un bilan des compétences, s'orienter ou se réorienter, etc. Comme l'observe le psychologue du travail Christophe Dejours dans les univers du travail, les individus sont eux aussi demandeurs d'une évaluation de leur travail: «La plupart de ceux qui travaillent souhaitent l'évaluation. Elle porte en elle la possibilité de donner un retour à celui qui travaille sur l'utilité et la qualité de ce qu'il donne de lui-même» (Dejours, 2003, p. 49). "Cette dimension, celle qui nous fait travailler, s'exprime sous une forme majeure: la reconnaissance. [...] Travailler, ce n'est plus seulement produire, c'est aussi se transformer soi-même. Le ressort fondamental de la mobilisation dans le travail, c'est l'attente du sujet par rapport à l'accomplissement de soi. C'est un moteur extrêmement puissant» (Ibid., p. 52). Dans le contexte de l'enseignement supérieur, des enseignants présents aux $1^{\text {res }}$ Assises de la formation (5 mars 2009, Montpellier SupAgro) ont 
souligné l'effet «attractif» de l'évaluation des compétences en situation professionnelle sur les étudiants. Lévy-Leboyer (2011) souligne que la principale attente des salariés envers l'évaluation au travail concerne son «équité».

Cette attente des personnes doit toutefois être nuancée, comme le fait Dejours lui-même. Elle se heurte à une autre logique à l'œuvre sur les milieux de travail: la tentation du secret. «Bien travailler, c'est toujours faire des infractions» (Dejours, 2003, p. 18). Or, «les savoir-faire, les trucs, les ficelles de métier constituent des leviers importants dans la négociation du pouvoir à l'intérieur d'une organisation. Quand bien même il n'y aurait pas de risque de sanction, l'agent a souvent intérêt à garder secrètes ses habiletés pour négocier sa place, sa qualification, son utilité pour l'organisation» (Loc. cit.). De plus, les risques de sanctions liées à certaines pratiques d'évaluation provoquent un sentiment de menace pour les individus, ce qui peut les amener à rejeter l'évaluation, du moins à la craindre suffisamment pour ruser avec elle. «Les travailleurs s'efforcent parfois de faire impression sur l'observateur ou l'évaluateur. Ils utilisent pour cela une mise en scène de leurs efforts » (Ibid., p. 25). Patton, un auteur nordaméricain qui a travaillé sur l'évaluation des programmes, constate le même phénomène, qu'il appelle «le syndrome de stress préévaluatif» : «Le désir d'être efficace est si fort que c'est souvent la source de l'anxiété évaluative» (Patton, 2008, p. 47).

\section{Les effets pervers}

Au-delà des effets attendus, certains chercheurs en sciences de l'éducation (Perrenoud, 2010; Baillé, 2001) ont souligné les effets pervers possibles du développement tous azimuts de pratiques d'évaluation des compétences. La non-prise en compte du travail réel dans sa complexité, le mythe d'une activité humaine pleinement rationnelle ainsi que le contrôle sur l'ensemble des tâches pour accroître la performance et la rentabilité amènent le plus souvent les personnes évaluées à adopter des stratégies de protection, de ruse ou d'évitement, lesquelles peuvent paralyser le développement professionnel (Perrenoud, 2010). L'évaluation semble ainsi prise en étau entre une fonction de contrôle et de normalisation et une fonction de reconnaissance et d'aide au développement, les deux étant toutefois liées. 
Baillé dénonce la «frénésie évaluative» qui touche l'école et l'entreprise. «L'usage du mot compétence dans la logomachie managériale et sa récupération par les pédagogues s'accordent à cette injonction paradoxale d'accroître l'autonomie du sujet en le soumettant à d'incessantes évaluations» (Baillé, 2001, p. 52). L'élève doit trouver en lui, comme l'esclave Ménon, toutes les ressources à son développement. On attend de lui «une plus forte implication (responsabilité et culpabilité) individuelle». «Dans les deux cas, entreprise et école, l'adhésion aux normes sociales suppose que les attributions de compétences s'attachent à la valorisation des attributions causales internes » (Loc. cit.). Le risque de telles pratiques est de renvoyer à l'individu seul et seulement à lui la responsabilité de ses compétences, au détriment de la prise en compte des conditions, des contextes et des situations de travail et d'enseignement qu'il a rencontrés (et parfois subis). Le risque est donc grand de naturaliser ou de trop personnaliser les compétences.

\section{Conclusion}

Cette revue de littérature nous a permis de dégager un certain nombre d'enseignements de la littérature sur l'évaluation des compétences professionnelles en évaluation, en éducation et en psychologie du travail, incluant la didactique professionnelle. Ces enseignements mettent en lumière les multiples situations où des compétences peuvent être évaluées, de manière directe et indirecte et pour plusieurs finalités.

Le premier enseignement est que l'évaluation des compétences est une injonction plutôt récente, mais qu'elle n'est pas si éloignée de ce qu'un certain nombre d'acteurs du monde éducatif pratiquent déjà, même si les termes changent. Elle invite en tout cas à remettre un certain nombre de pratiques «sur le métier», comme le dit Perrenoud (2004), et à repenser l'articulation entre connaissances et compétences.

Le deuxième enseignement est qu'un consensus semble se dessiner, aussi bien en sciences de l'éducation qu'en psychologie du travail, autour de l'idée que les compétences professionnelles sont liées aux situations. Il semble donc possible, aujourd'hui, de s'accorder sur une définition et d'en tirer certaines implications pratiques. Si les compétences se voient à la capacité de quelqu'un à agir sur et dans des situations variées, leur évaluation implique un détour nécessaire - mais non suffisant - par l'activité (de travail 
ou scolaire) en situation réelle, que celle-ci se déroule sous les yeux de l'observateur ou qu'elle soit passée puis racontée et reconstituée. L'évaluation devrait même, en théorie, concerner plusieurs situations.

Également, les risques de l'évaluation des compétences en milieu scolaire et professionnel sont mieux connus. Plusieurs auteurs soulignent que, dans ce détour par l'activité en situation, l'évaluateur peut être tenté d'en rester au visible, au mesurable, au chiffrable ou au procédural, au détriment d'une prise en compte plus globale de tout ce qui fait la complexité et la richesse de l'activité et des situations qui la déterminent, notamment des dimensions cognitives et conceptuelles de l'activité. L'évaluateur court ainsi le risque de devoir s'ouvrir au réel de l'activité de travail (Clot, 2008) et d'y réviser une partie de ses certitudes.

Le quatrième enseignement porte sur l'activité des évaluateurs, qui est désormais mieux connue. Les principaux critères et indicateurs de compétences qu'ils prélèvent et analysent sont identifiés, même si leur désignation n'est pas stabilisée. Le rôle et les limites des référentiels sont également mieux connus, ainsi que les processus dynamiques qui gouvernent cette activité et les risques de biais qu'elle soulève.

Enfin, les effets possibles, positifs ou pervers, de ce type de pratique, dont la technicité ne doit pas faire croire à la neutralité, comme l'ont souligné plusieurs auteurs, sont également mieux connus.

Si évaluer des compétences, c'est évaluer un objet mouvant et difficile d'accès, comme nous venons de le voir, ceci n'implique-t-il pas de redoubler de précautions pour ne pas être victime d'une volonté de mesure mortifère? Faut-il encourager les évaluateurs à faire le «deuil du vrai» dont parlait Michel Vial (2001)? Ne faut-il pas les encourager à ruser, comme le dit le sociologue Pierre Merle (2007), avec la complexité d'une situation pédagogique ou professionnelle insérée dans un flux de relations dont il faut tenir compte pour assurer la poursuite par de multiples «arrangements»? Ne faut-il pas, comme le dit Christophe Dejours (2003), rappeler aux évaluateurs de se contraindre à toujours tenir compte des autres, des personnes évaluées, pour qui l'évaluation est un jeu à enjeux, risqué et exposant, qu'il s'agit de ne pas pervertir au nom d'une «vérité» ou d'une «efficacité» illusoires? Toute évaluation des compétences ne devrait-elle pas être entourée de principes déontologiques, comme ceux qui sont cités par Lévy-Leboyer: «intégrité», «responsabilité professionnelle», «respect des autres et de la dignité», «souci du bien-être des autres» et «responsabilité sociale» (Lévy-Leboyer, 2011, p. 14)? 


\section{NOTES}

1. Ces recherches s'intéressent aussi bien à l'évaluation des compétences professionnelles en formation, sur les lieux de travail et dans le cadre de la validation des acquis de l'expérience.

2. Impliquées dans la réalisation de tâches scolaires (voir Rey, 2014).

3. Theureau (1994) rappelle que le concept a été introduit plutôt récemment en ergonomie, dans les années 1980, non sans susciter quelque méfiance, et qu'il est venu remplacer celui de conduite, qui était davantage utilisé auparavant. Il soulignait en 1994 que, malgré les nombreuses tentatives, la compétence restait un concept instable où de nombreuses inconnues demeuraient.

4. Le BIEF est un organisme de conseil et d'intervention reconnu à l'échelle internationale, et a été fondé par des chercheurs belges: De Ketele et Roegiers. Il publie des ressources en ligne. Voir son site web: www.bief.be.

5. La tentation est grande de faire des compétences une substance, un trait fixe qui appartiendrait à l'individu. Et ainsi, comme le dit Dejours, de «glisser d'une évaluation du travail à une évaluation de la personne» (Dejours, 2003, p. 36).

6. Voir par exemple l'auteur américain Patton, en évaluation de programme, qui cite le biais de confirmation mis à jour en psychologie sociale et son rôle possible dans l'activité des évaluateurs: «La tendance à chercher des preuves pour confirmer nos préconceptions et nos biais» (Patton, 2008, p. 48).

7. Toute pratique d'autoévaluation de ses compétences doit-elle être alors évitée? Pas nécessairement, car, comme l'ont montré d'autres études inspirées des travaux de Bandura (voir Nagels, 2010), le sentiment de compétence ou «sentiment d'autoefficacité» peut être un élément important à prendre en compte dans l'évaluation, à condition de le considérer pour ce qu'il est: un ensemble de «croyances » dont le lien avec le niveau effectif du sujet doit être mis en question. 


\section{RÉFÉRENCES}

Allal, L., \& Mottier Lopez, L. (2009). Au cœur du jugement professionnel en évaluation: des démarches de triangulation. Les dossiers des sciences de l'éducation, 22, $25-40$.

Anderson, J. R. (1982). Acquisition of cognitive skill. Psychological Review, 89(4), 369-406. doi: 10.1037/0033-295X.89.4.369

Baillé, J. (2001). Les compétences, de nouveaux «objets» pour l'évaluateur. Dans G. Figari \& M. Achouche (dir.). L'activité évaluative réinterrogée: regards scolaires et socioprofessionnels (pp. 52-67). Bruxelles: De Boeck.

Cardinet, J. (2003). Faut-il parler d'édumétrie? Bulletin de l'Association pour le développement des méthodologies de l'évaluation en éducation, ADMEE-Europe, 2002(3) et 2003(1).

Chenu, F. (2012). L'évaluation des compétences: de la méthodologie de la réflexivité garante de la transférabilité aux perspectives ouvertes par la didactique professionnelle (Thèse de doctorat). Université de Liège, Belgique.

Clot, Y. (2008). Travail et pouvoir d'agir. Paris: PUF.

Crahay, M. (2006). Dangers, incertitudes et incomplétude de la logique de la compétence en éducation. Revue française de pédagogie, 154, 97-110.

Crahay, M. (2014). L'évaluation des compétences: un nouveau défi lancé aux sciences de l'éducation? Dans C. Dierendonck, E. Loarer, \& B. Rey (dir.). L'évaluation des compétences en milieu scolaire et professionnel (chap. 12). Bruxelles: De Boeck.

De Ketele, J.-M. (2001). Place de la notion de compétence dans l'évaluation des apprentissages. Dans G. Figari \& M. Achouche (dir.). L'activité évaluative réinterrogée: regards scolaires et socioprofessionnels (pp. 39-43). Bruxelles: De Boeck Université.

De Montmollin, M. (1986). L'intelligence de la tâche: éléments d'ergonomie cognitive. Berne: Peter Lang.

Dejours, C. (2003). L'évaluation du travail à l'épreuve du réel: critique des fondements de l'évaluation. Paris: INRA Éditions.

Dierendonck, C., Loarer, E., \& Rey, B. (2014). L'évaluation des compétences en milieu scolaire et professionnel. Bruxelles: De Boeck.

Dreyfus, M. L., Dreyfus, S. E., \& Athanasiou, T. (1986). Mind over machine: The power of human intuition and expertise in the era of the computer. New York: The Free Press.

Dujarier, M. A. (2006). L'idéal au travail. Paris: PUF.

Engeström, Y., \& Sannino, A. (2010). Studies of expansive learning: Foundations, findings and future challenges. Educational Research Review, 5, 1-24. doi:10.1016/ j.edurev.2009.12.002

Figari, G. (1994). Évaluer : quel référentiel? Bruxelles: De Boeck.

Gérard, F. M. (2005, octobre). L'évaluation des compétences à travers les situations complexes. Actes du $18^{\mathrm{e}}$ Colloque international de l'ADMEE-Europe, Reims (France). 
Jonnaert, P. (2014). Évaluer des compétences? Oui, mais de quelles compétences s'agitil? Dans C. Dierendonck, E. Loarer, \& B. Rey (dir.). L'évaluation des compétences en milieu scolaire et professionnel (chap. 2). Bruxelles: De Boeck.

Jonnaert, P., Barrette, J., Masciotra, D., \& Yaya, M. (2006, août). La compétence comme organisateur des programmes de formation revisitée, ou la nécessité de passer de ce concept à celui de "l'agir compétent». Actes du Colloque de l'Observatoire des réformes en éducation, Montréal (Canada).

Jorro, A. (2010). Le développement professionnel des acteurs : une nouvelle fonction de l'évaluation? Dans L. Paquay, C. Van Nieuwenhoven \& P. Wouters (dir.). L'évaluation, levier du développement professionnel? (pp. 253-264). Bruxelles: De Boeck.

Lacourse, F., \& Oubenaïssa-Giardina, L. (2009). L'ePortfolio de compétences professionnelles: de la reprise réflexive à l'évaluation. Les dossiers des sciences de l'éducation, 22, 41-55.

Le Boterf, G. (2006). Ingénierie et évaluation des compétences. Paris: Éditions d'Organisation.

Lebel, C. (2009). L'interprétation d'un référentiel en situation d'évaluation. Les dossiers des sciences de l'éducation, 22, 41-51.

Leplat, J. (1997). Regards sur l'activité en situation de travail: contribution à la psychologie ergonomique. Paris: PUF.

Lévy-Leboyer, C. (2011). Évaluation du personnel. Paris : Éditions d'Organisation.

Loarer, E. (2014). Compétence et évaluation en milieu professionnel: une relation complexe. Dans C. Dierendonck, E. Loarer \& B. Rey (dir.). L'évaluation des compétences en milieu scolaire et professionnel (chap. 13). Bruxelles: De Boeck.

Loarer, E., \& Pignault, A. (2010). Analyser les acquis de l'expérience professionnelle des personnes faiblement qualifiées: quels repères méthodologiques? Dans L. Paquay, C. Van Nieuwenhoven \& P. Wouters (dir.). L'évaluation, levier du développement professionnel? (pp. 209-224). Bruxelles: De Boeck.

Mayen, P. (2001). Thèse pour l'habilitation à diriger des recherches en sciences de l'éducation, Développement professionnel et formation: une théorie didactique (Thèse de doctorat). Université Pierre-Mendès-France, Grenoble.

Mayen, P. (2005, mai). L'évaluation des compétences: un pari sur l'avenir. Actes du Séminaire des responsables de formation CNFEPJJ, Vaucresson, France. Récupéré de http://194.199.119.55/site/images/mesure_competence.pdf

Mayen, P. (2009). Expérience et formation des adultes. Dans J.-M. Barbier, E. Bourgeois, J.-C. Ruano-Borbalan \& G. Chapelle (dir.). Encyclopédie de la formation (pp. 763-780). Paris: PUF.

Mayen, P., Mayeux, C., \& Savoyant, A. (2006). Construire la référence: une activité permanente des jurys de VAE. Dans G. Figari, P. Rodrigues, M. Palmira Alves \& P. Valois (dir.). Évaluation des compétences et apprentissages expérientiels: savoirs, modèles et méthodes (pp. 126-143). Lisbonne: ADMEE-Europe et Educa.

Mayen, P., Métral, J.-F., \& Tourmen, C. (2010). Les situations de travail: références pour les référentiels. Recherche et formation, 64, 31-46. 
Mayen, P., \& Vanhulle, S. (2010). Quels repères de développement dans les situations de travail? Dans L. Paquay, C. Van Nieuwenhoven \& P. Wouters. (2010). L'évaluation, levier du développement professionnel? (pp. 225-237). Bruxelles: De Boeck.

Merle, P. (2007). Les notes: secrets de fabrication. Paris: PUF.

Métral, J.-F., Tourmen, C., \& Mayen, P. (2014). Évaluer les compétences par l'activité en situation. Dans C. Dierendonck, E. Loarer, \& B. Rey (dir.). L'évaluation des compétences en milieu scolaire et professionnel (chap. 16). Bruxelles: De Boeck.

Nagels, M. (2010). Construire l'auto-efficacité par l'analyse de l'activité en formation des cadres et dirigeants de santé publique. Savoirs, 1(22), 69-88.

Paquay, L., Van Nieuwenhoven, C., \& Wouters, P. (2010). L'évaluation, levier du développement professionnel? Bruxelles: De Boeck.

Parry, S. B. (1997). Evaluating the impact of training: A collection of tools and techniques. American Society for Training and Development: Alexandria (VA).

Patton, M. Q. (2008). Utilization-focuzed evaluation (4th ed.). Sage: Los Angeles (CA).

Perrenoud, P. (2004). Évaluer des compétences. L'éducateur, $n^{\circ}$ spécial « La note en pleine évaluation », 8-11.

Perrenoud, P. (2010). Et si l'évaluation institutionnelle paralysait le développement professionnel? Dans L. Paquay, C. Van Nieuwenhoven \& P. Wouters (dir.). L'évaluation, levier du développement professionnel? (pp. 37-48). Bruxelles: De Boeck.

Piaget, J. (1947). Psychologie de l'intelligence. Paris : Colin.

Rey, B. (1996). Les compétences transversales en question. Paris : ESF.

Rey, B. (2014). Compétence et évaluation en milieu scolaire: une relation complexe. Dans C. Dierendonck, E. Loarer \& B. Rey (dir.). L'évaluation des compétences en milieu scolaire et professionnel (chap. 1). Bruxelles: De Boeck.

Roegiers, X. (2001). Une pédagogie de l'intégration: compétences et intégration des acquis dans l'enseignement. Bruxelles: De Boeck.

Roegiers, X. (2014). Quelle évaluation des compétences au service de quel projet pour l'école? Dans C. Dierendonck, E. Loarer \& B. Rey (dir.). L'évaluation des compétences en milieu scolaire et professionnel (chap. 4). Bruxelles: De Boeck.

Serres, C. (2014). Avant-propos. Dans C. Dierendonck, E. Loarer \& B. Rey (dir.). L'évaluation des compétences en milieu scolaire et professionnel. Bruxelles: De Boeck.

Stroobants, M. (1994). La visibilité des compétences. Dans F. Rope \& L. Tanguy (dir.). Savoirs et compétences: de l'usage de ces notions dans l'école et l'entreprise. Paris : L'Harmattan.

Tardif, J., \& Dubois, B. (2010). Construire des dispositifs en vue de l'évaluation et du développement des compétences: comment? Dans L. Paquay, C. Van Nieuwenhoven \& P. Wouters. (dir.). L'évaluation, levier du développement professionnel? (pp. 133-146). Bruxelles: De Boeck.

Theureau, J. (1994). Compétences et ergonomie. Dans M. De Montmollin (dir.). Sur le travail: choix de textes (pp. 1-7. Toulouse: Octarès.

Tourmen, C. (2009). L'activité évaluative et la construction progressive du jugement. Les dossiers des sciences de l'éducation, 22, 101-119. 
Tourmen, C. (2012, janvier). Comment voir la pensée dans l'action? Actes du 24 Colloque international de l'ADMEE-Europe, Luxembourg. Récupéré de http:// admee2012.uni.lu/pdf2012/S7_04.pdf

Tourmen, C., Deblay, S., Lipp, A., \& Boude, A. (2013). Évaluer en situation professionnelle. Rapport de recherche remis au ministère de l'Agriculture, de l'Agroalimentaire et de la Forêt, France.

Tourmen, C, \& Mayen, P. (2012). Les évaluateurs, des épistémologues en actes? Raisons éducatives, 16, 63-75. doi: 10.3917/dbu.lopez.2012.01.0063

Tourmen, C., Mayen, P., \& Figari, G. (2009, janvier). Quels critères et indicateurs pour évaluer les acquis de l'expérience? Actes du 21 Colloque international de l'ADMEE-Europe, Louvain-La-Neuve (Belgique). Récupéré de http://admee.webfactional.com/Plone/symposiums/premiers-auteurs-en-p/presse-m.-ch-1/S-07.3Tourmen $\% 20$ C.pdf

Valsiner, J. (2007). Culture in minds and societies. Sage: New Delhi.

Vergnaud, G. (1990). La théorie des champs conceptuels. Recherches en didactique des mathématiques, 10(2-3), 133-170.

Vergnaud, G. (2001). Psychologie du développement cognitif et évaluation des compétences. Dans G. Figari \& M. Achouche (dir.). L’activité évaluative réinterrogée: regards scolaires et socioprofessionnels (pp. 43-51). Bruxelles: De Boeck Université.

Vial, M. (2001). Évaluation et régulation. Dans G. Figari \& M. Achouche (dir.). L’activité évaluative réinterrogée (pp. 68-78). Bruxelles: De Boeck.

Vygotski, L. S. (1934/1997). Pensée et langage. Paris: Éditions Sociales.

Zarifian, P. (2001). Le modèle de la compétence. Paris : Liaisons. 